\title{
Hypophosphatemia Associated with Intravenous Iron Therapies for Iron Deficiency Anemia: A Systematic Literature Review
}

This article was published in the following Dove Press journal:

Therapeutics and Clinical Risk Management

John A Glaspy'

Michelle Z Lim-Watson (iD) ${ }^{2}$

Michael A Libre $\mathbb{D}^{3}$

Swagata S Karkare ${ }^{3}$

Nandini Hadker ${ }^{3}$

Aleksandra Bajic-Lucas (iD) ${ }^{2}$

William E Strauss ${ }^{2}$

Naomi $\vee$ Dahl (iD ${ }^{2}$

'UCLA School of Medicine, Los Angeles, CA, USA; ${ }^{2}$ AMAG Pharmaceuticals, Inc., Waltham, MA, USA; ${ }^{3}$ Trinity LifeSciences, Waltham, MA, USA
Correspondence: Naomi $\vee$ Dahl AMAG Pharmaceuticals, Inc., II 100 Winter Street, Waltham, MA 0245I, USA $\mathrm{Tel}+$ I 617-498-7650

Email ndahl@amagpharma.com
Background: Iron deficiency anemia (IDA) is a prevalent yet underdiagnosed condition with a significant impact on quality of life. Oral iron supplementation is often poorly tolerated or yields inadequate response, requiring the use of intravenous iron (IVI) in some patients. Administration of certain IVI preparations has been associated with decreases in serum phosphate levels and clinically significant hypophosphatemia, which has been reported to lead to adverse events including serious fatigue and osteomalacia.

Objective: The purpose of this study was to systematically assess the prevalence, clinical consequences, and reporting of treatment-emergent hypophosphatemia within literature investigating IVI therapies marketed in the United States (US).

Methods: A systematic literature review (SLR) was conducted using the PubMed database to identify publications reporting serum phosphate levels or rates of hypophosphatemia within adult IDA patient populations receiving current US-marketed IVIs.

Results: The SLR yielded 511 unique publications, with 40 records meeting the final inclusion criteria. Most studies did not report phosphate monitoring methodology or an explicit definition of hypophosphatemia. Hypophosphatemia rates ranged from $0.0 \%$ to $92.1 \%$ for ferric carboxymaltose (FCM), $0.0 \%$ to $40.0 \%$ for iron sucrose, $0.4 \%$ for ferumoxytol, and $0.0 \%$ for low-molecular-weight (LMW) iron dextran. Randomized controlled studies described hypophosphatemia as "asymptomatic" or did not report on other associated sequelae. Eleven case reports detailed treatment-emergent hypophosphatemia in patients treated with FCM. Patients with acute hypophosphatemia primarily developed severe fatigue; those with repeated FCM dosing developed chronic hypophosphatemia associated with osteomalacia and bone deformities.

Conclusion: Studies analyzed in this SLR reported a range of hypophosphatemia rates, with the highest consistently seen in patients treated with FCM. Across the clinical literature, there appeared to be minimal standardization of phosphate monitoring and definitions of hypophosphatemia. Although multiple cases have documented serious clinical consequences of hypophosphatemia associated with certain IVIs, current trials neither consistently nor adequately assess the frequency and severity of treatment-emergent hypophosphatemia and may underestimate its prevalence.

Keywords: IDA, phosphate, hypophosphatemia, iron supplementation

\section{Plain Language Summary}

Iron deficiency anemia (IDA) is a common and debilitating condition that may negatively impact a patient's quality of life. Although oral iron supplementation is the most common treatment for IDA, some patients may have poor tolerance, response, or compliance with 
oral iron. Intravenous iron (IVI) therapies have thus become increasingly common and are becoming preferred treatments for many patients with iron deficiency anemia (IDA). Although newer generation IVIs are broadly effective and safe, an increasing number of case reports and clinical trials have recently associated certain IVIs with treatment-emergent hypophosphatemia (abnormally low serum phosphate). This literature review analyzed the prevalence, clinical consequences, and reporting of treatment-emergent hypophosphatemia within the clinical literature investigating US-marketed IVI therapies. This review identifies significant inconsistency across the clinical literature in the reporting of serum phosphate monitoring, definitions and rates of treatment-emergent hypophosphatemia. Current IVI studies likely significantly underestimate the prevalence and consequences of hypophosphatemia due to lack of trials designed to systematically identify and monitor changes in serum phosphate.

\section{Introduction}

Iron deficiency anemia (IDA) is a prevalent, yet underdiagnosed condition with significant clinical and quality of life impact on patients. ${ }^{1}$ Symptoms of IDA include chronic weakness, fatigue, difficulty concentrating and exercise intolerance, resulting in decreased productivity. ${ }^{2}$ IDA affects an estimated five million adults in the United States (US), and globally represents the fifth leading cause of years lived with disability. ${ }^{3,4}$ Common etiologies of IDA include chronic inflammatory diseases or conditions leading to blood loss or malabsorption of iron such as hypermenorrhea or abnormal uterine bleeding (AUB), pregnancy, inflammatory bowel disease (IBD), chronic kidney disease (CKD), chronic heart failure, cancer, and bariatric surgery. ${ }^{2}$

Due to poor tolerability, efficacy and compliance with oral iron supplementation, intravenous iron (IVI) has become an increasingly utilized therapeutic option in the treatment of IDA. ${ }^{5-8}$ Research suggests that IVIs are better tolerated than oral iron and provide a faster onset of action due to the much more rapid correction of body iron stores. Certain IV formulations also allow the administration of an entire therapeutic iron dose in a single sitting, which may be more convenient and improve patient compliance. ${ }^{9-11}$ Although older IVI preparations such as high-molecularweight (HMW) iron dextran have been associated with potentially serious adverse events such as hypersensitivity reactions, newer IVI formulations including iron sucrose, ferric gluconate, low-molecular-weight (LMW) iron dextran, ferric carboxymaltose (FCM), and ferumoxytol have been generally shown to be safer. ${ }^{12-15}$
A growing number of case reports have described treatment-emergent hypophosphatemia (abnormally low serum phosphate) following IVI administration as a safety consideration. ${ }^{16-18}$ In the acute setting, hypophosphatemia may cause rhabdomyolysis, arrhythmias, and respiratory failure; chronic hypophosphatemia has been associated with osteomalacia, bone deformities, and increased inpatient mortality. ${ }^{19,20}$ Although hypophosphatemia may have serious clinical consequences, its diagnosis may be commonly missed in the clinic due to initial nonspecific symptomatic presentation as generalized weakness and fatigue. $^{21}$

To the best of our knowledge, a systematic investigation of hypophosphatemia and its downstream clinical consequences across all US-marketed intravenous iron therapies has not been conducted to date. ${ }^{2,22}$ This systematic literature review (SLR) was hence designed to analyze the prevalence, clinical consequences, and reporting of treatment-emergent hypophosphatemia within the clinical literature investigating IVI therapies marketed in the US.

\section{Methods}

\section{Literature Search Methodology}

An SLR was conducted in PubMed in accordance with the Preferred Reporting Items for Systematic Reviews and MetaAnalyses (PRISMA) guidelines to identify publications reporting on current US-marketed IVIs within adult IDA patient populations of any etiology. English-language articles published within 10 years of the search date $(2 / 28 / 2019)$ were included in the literature review. Search terms (Appendix A) for IDA, as well as generic and trade names of all US-marketed IVIs, were used to index all possible literature for subsequent screening by reviewers. At the time of the literature search, US-marketed IVIs included LMW iron dextran $\left(\mathrm{INFeD}^{\circledR}\right.$, CosmoFer $\left.^{\circledR}\right)$, ferric gluconate $\left(\right.$ Ferrlecit $\left.^{\circledR}\right)$, ferric carboxymaltose (Injectafer ${ }^{\circledR}$, Ferinject ${ }^{\circledR}$ ), ferumoxytol $\left(\right.$ Feraheme $\left.^{\circledR}\right)$, and iron sucrose (Venofer $\left.{ }^{\circledR}\right)$.

\section{Study Selection}

Two reviewers independently screened all studies identified in the initial literature search based on previously established inclusion and exclusion criteria. Studies were included if they represented original research, investigated US-marketed IVI therapies, and reported serum phosphate levels (even if not classified as hypophosphatemia explicitly) and/or hypophosphatemia rates (including literature citing $0.0 \%$ rates, if reported). If studies met these 
requirements, they were included, regardless of the frequency with which phosphate levels were collected. Studies that did not report either of these endpoints were excluded. Studies were also excluded if they met any of the following criteria: (a) duplicates, letters, commentaries, or reviews; (b) investigated non-US marketed IVI therapies exclusively; (c) focused on pediatric populations; or (d) included only hemodialysis patients (excluded due to these patients' inability to excrete phosphate and potential confounding use of phosphate binders). Study subgroups or treatment arms that fulfilled the screening criteria were included even if other treatment arms or subgroups within that same study did not. For example, studies reporting separately on patients with non-dialysis and dialysis-dependent chronic kidney disease (CKD) were broken out to only include the non-dialysis dependent CKD (NDD-CKD) patient arm of the study.
Publications meeting all screening criteria were stratified by level of evidence (Figure 1). Level I evidence included randomized controlled trials (RCTs), Level II evidence included observational, retrospective, or post hoc studies, and Level III evidence included case reports.

\section{Data Extraction}

Two reviewers independently screened and extracted data from each publication that met the inclusion criteria. Data included study sample demographics, methodology (including dosing schedule, serum phosphate measurement, and follow-up protocol), as well as reported rates and clinical consequences of hypophosphatemia. Relevant reporting specifications, including definition of hypophosphatemia (when indicated) and serum phosphate reference ranges used were collected. We relied solely upon the publications cited in our analysis; we did not contact the authors or

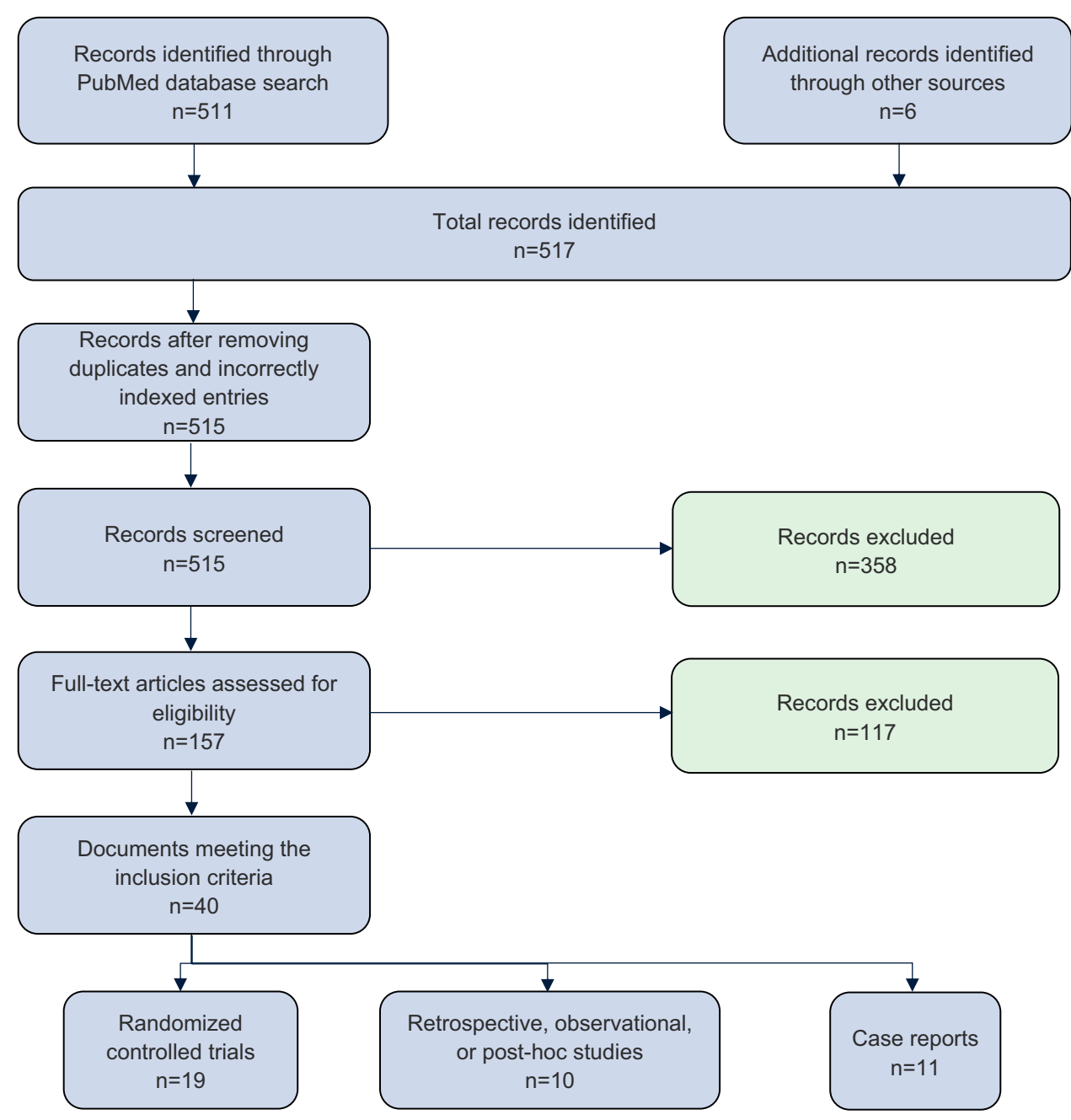

Figure I PRISMA flow diagram detailing SLR record screening for hypophosphatemia in adult IDA patients receiving US-marketed IVI therapies. Abbreviations: SLR, systematic literature review; IDA, iron deficiency anemia; US, United States; IVI, intravenous iron. 
journals to seek unpublished information. Rates of hypophosphatemia were recorded using definitions of hypophosphatemia presented within the individual study. All data analysis was performed using Microsoft Excel. This review was not considered appropriate for a meta-analysis due to the heterogeneity of patient populations, IVI dosing regimens, phosphate measurement methodologies, and inconsistent definitions of hypophosphatemia reported across studies.

\section{Results}

The PubMed literature search yielded 511 unique publications. Additional searches in PubMed and bibliographies of review articles yielded 6 case reports which were added to our literature pool. After removing duplicates and misindexed publications, 515 records remained for screening. Abstract screening removed 358 records. Two independent reviewers screened 157 full-text publications, excluding 117 additional records. Reasons for exclusion included sole evaluation of IVI therapies marketed outside of the US exclusively, lack of reporting of hypophosphatemia or serum phosphate levels as an endpoint, and focus on pediatric, non-hemodialysis or non-IDA patient populations (Appendix B presents a complete summary of the excluded publications). A total of 40 records across the 3 evidence levels met the final inclusion criteria. A PRISMA-compliant summary of the literature search and screening process is included in Figure 1.

\section{Definitions and Reporting of Hypophosphatemia}

Within the literature reviewed, 13 of the 19 Level I and 4 of the 10 Level II studies did not report a definition of hypophosphatemia. ${ }^{9,23-38}$ Methodologies for serum phosphate measurement and clinical definition of hypophosphatemia varied within and across Level I and Level II studies. Of the 19 Level I studies, 15 did not explicitly report the methodology or timing of serum phosphate measurement. Three of the 10 Level II studies simply used any serum phosphate measurement pre- and post-administration. ${ }^{17,36,38}$ Serum phosphate measurement in RCTs likely occurred during hematological evaluations; however, only 5 studies explicitly reported serum phosphate measurement. ${ }^{26,30,39-41}$ In 2 studies, only laboratory abnormalities deemed "clinically significant" were recorded by study investigators. ${ }^{25,26}$ One such study by Breymann et al (2017) reported phosphate levels below $0.6 \mathrm{mmol} / \mathrm{L}(2 \mathrm{mg} / \mathrm{dL})$-the Common
Terminology Criteria for Adverse Events (CTCAE) threshold for "severe" hypophosphatemia - at week 3 in 11 women (10 from the FCM arm and 1 from the comparator oral ferrous sulphate arm). ${ }^{31}$ However, this study did not consider these depressed phosphate levels to be clinically relevant as phosphate levels in patients recovered by the end of the study. More importantly, this study did not record this finding as a hypophosphatemic treatment-emergent adverse event. In contrast, Prats et al (2013) classified NDD-CKD patients enrolled in their study as either hypophosphatemic or non-hypophosphatemic if phosphate levels decreased or remained unchanged or even increased compared to baseline levels at week 3 post-treatment. ${ }^{42}$ In others, the timing of hematological evaluation was not explicitly reported by investigators. ${ }^{9,30}$

Standard guidelines advocated by the CTCAE designate hypophosphatemia as Grade 1 (mild) $(<\mathrm{LLN}-2.5 \mathrm{mg} /$ $\mathrm{dl} ;<\mathrm{LLN}-0.8 \mathrm{mmol} / \mathrm{l})$, Grade 2 (moderate) $(<2.5-2.0 \mathrm{mg} /$ $\mathrm{dl} ;<0.8-0.6 \mathrm{mmol} / \mathrm{l})$, Grade 3 (severe) $(<2.0-1.0 \mathrm{mg} / \mathrm{dl}$; $<0.6-0.3 \mathrm{mmol} / \mathrm{l}$ ), Grade 4 (Life-threatening consequences; urgent intervention indicated $)(<1.0 \mathrm{mg} / \mathrm{dl} ;<0.3$ mmol/l) and Grade 5 (Death). ${ }^{43}$ One RCT adopted the CTCAE "moderate" hypophosphatemia definition. ${ }^{29}$ Five RCTs used the CTCAE definition for "severe" hypophosphatemia. ${ }^{16,39-41,44}$ An additional study defined hypophosphatemia simply as any decrease in serum phosphate. $^{42}$

\section{Level I Evidence}

Literature screening identified 19 RCTs reporting on serum phosphate or hypophosphatemia., 16,23-34,39-41,44,45 Key characteristics of the identified studies are presented in Table 1. Of the 19 studies, 18 evaluated ferric carboxymaltose (FCM), 5 evaluated iron sucrose, and 1 each evaluated LMW iron dextran and ferumoxytol, respectively. Fourteen studies had sample sizes greater than 100 patients. $9,16,23-27,30-34,41,44,45$ Study patient populations spanned a variety of IDA etiologies and study timelines ranged in duration from 2 to 52 weeks. In most studies, FCM was administered as 1 or 2 doses of $750 \mathrm{mg}$ or $1000 \mathrm{mg}$; ferumoxytol was dosed in 2 doses of $510 \mathrm{mg}$ each; iron sucrose was dosed in multiple doses of $200 \mathrm{mg}$, and iron dextran was dosed via the Ganzoni formula: weight in $\mathrm{kg} \times(15$-current hemoglobin $\mathrm{g} / \mathrm{dL}) \times 2.4+$ $500=$ total iron requirement in $\mathrm{mg}$.

Rates of treatment-emergent hypophosphatemia varied significantly across RCTs (Figure 2). Hypophosphatemia rates ranged from $0.0 \%$ to $92.1 \%$ for $\mathrm{FCM}, 0.0 \%$ to $40.0 \%$ 


\begin{tabular}{|c|c|c|c|c|c|c|c|c|c|}
\hline 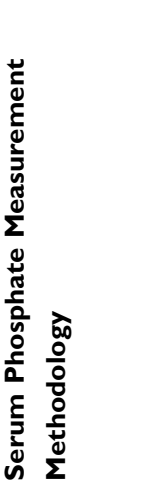 & 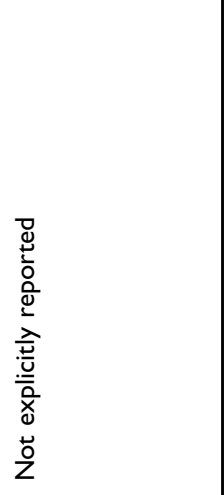 & 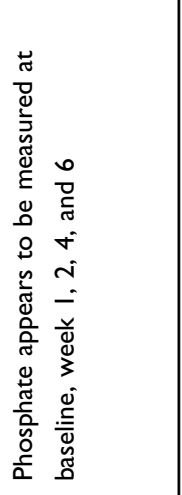 & 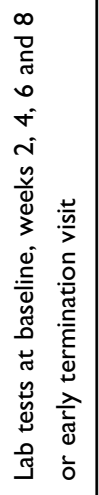 & 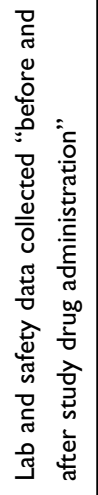 & 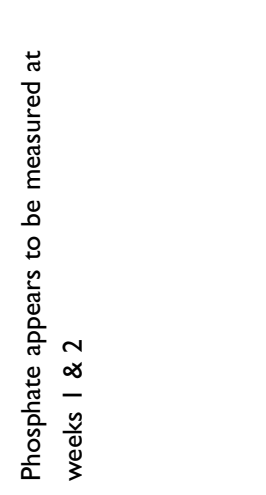 & \multicolumn{2}{|c|}{ 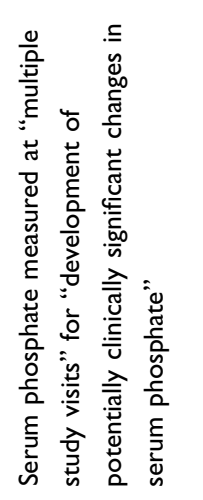 } & \multicolumn{2}{|c|}{ 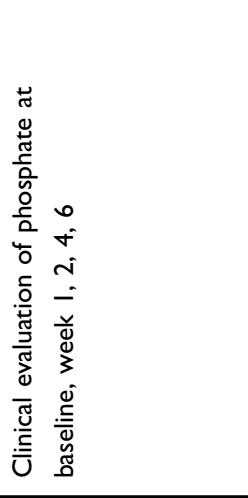 } \\
\hline 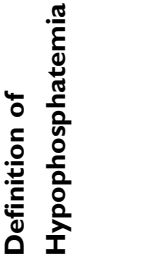 & 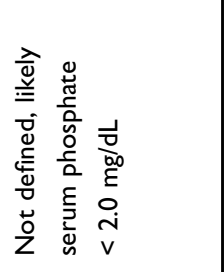 & 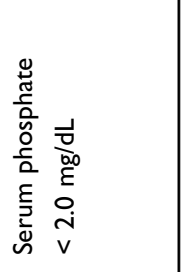 & 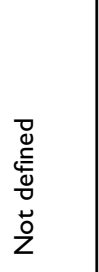 & 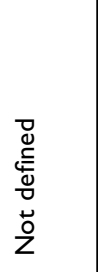 & 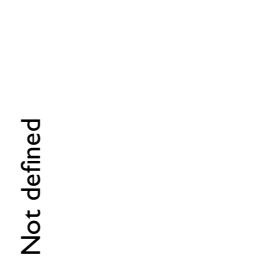 & \multicolumn{2}{|l|}{ 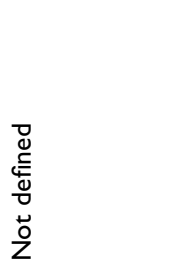 } & \multicolumn{2}{|c|}{ 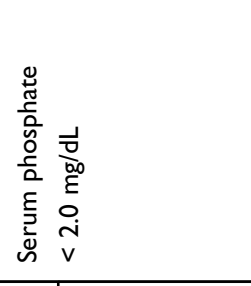 } \\
\hline 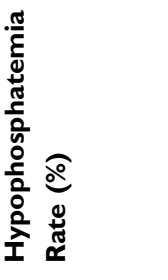 & 宂 & $\begin{array}{l}\stackrel{\circ}{\circ} \\
\text { ò }\end{array}$ & 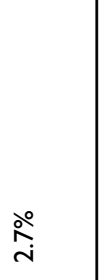 & $\stackrel{\stackrel{\circ}{\text { mे }}}{+}$ & $\begin{array}{l}\stackrel{\circ}{\circ} \\
\text { فे }\end{array}$ & $\begin{array}{l}\text { ○ें } \\
\text { ळo }\end{array}$ & 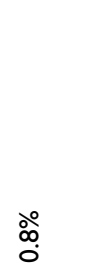 & 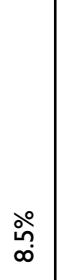 & ○ें \\
\hline 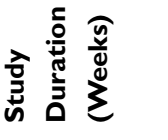 & in & 0 & $\infty$ & $\sigma$ & $\sim$ & \multicolumn{2}{|l|}{$\infty$} & \multicolumn{2}{|l|}{0} \\
\hline 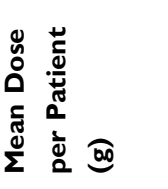 & 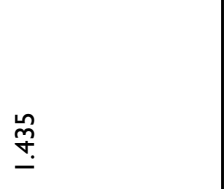 & 总 & $\stackrel{\infty}{\underline{\underline{x}}}$ & 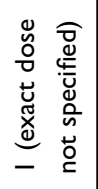 & $\begin{array}{l}\text { ऽ } \\
\circ \\
0\end{array}$ & 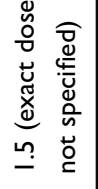 & 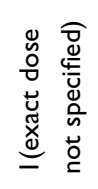 & $\stackrel{\varphi}{\underline{\varphi}}$ & $\stackrel{+}{m}$ \\
\hline 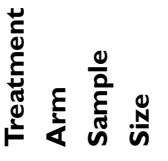 & $\stackrel{\stackrel{\alpha}{\alpha}}{\sigma}$ & $\stackrel{\infty}{\sim}$ & 全 & 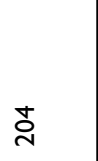 & 命 & 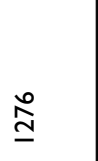 & 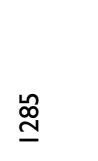 & $\tilde{\infty}$ & in \\
\hline $\begin{array}{l}\frac{0}{0} \\
\frac{0}{3} \\
\frac{\pi}{0} \\
\vdots \\
0\end{array}$ & 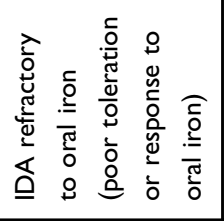 & 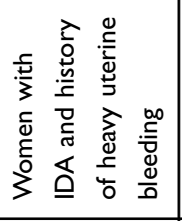 & 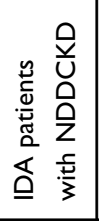 & 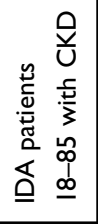 & 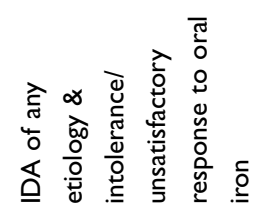 & \multicolumn{2}{|c|}{ 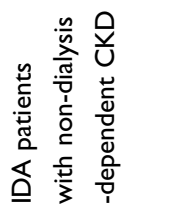 } & \multicolumn{2}{|c|}{ 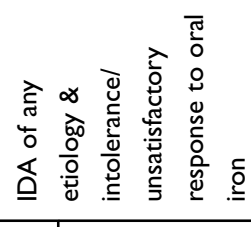 } \\
\hline 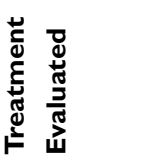 & $\sum_{\cup}$ & 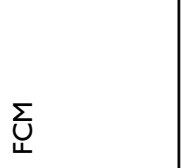 & $\sum_{\cup}$ & $\sum_{\cup}$ & $\sum_{U}$ & $\sum_{U}$ & 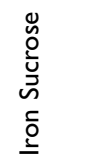 & $\sum_{U}$ & 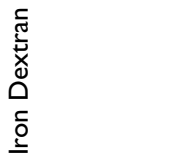 \\
\hline 旁 & 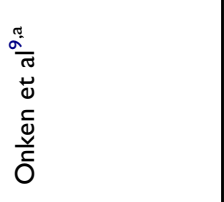 & 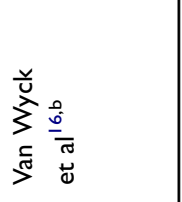 & 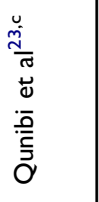 & 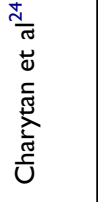 & 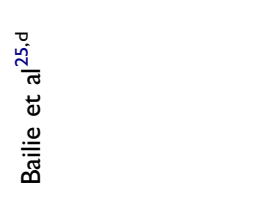 & \multicolumn{2}{|c|}{ 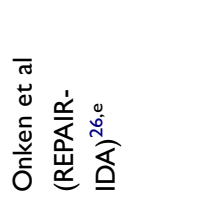 } & \multicolumn{2}{|l|}{ 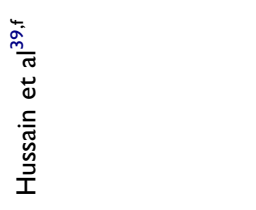 } \\
\hline
\end{tabular}




\begin{tabular}{|c|c|c|c|c|c|c|c|}
\hline 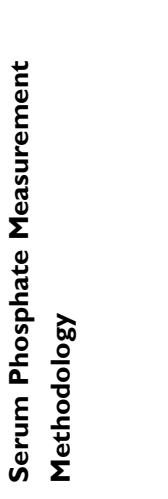 & 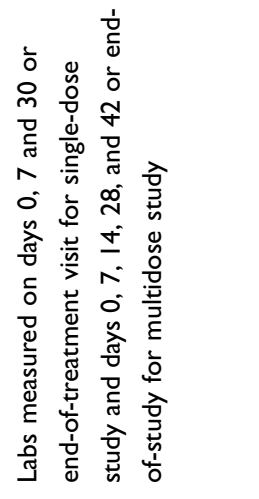 & 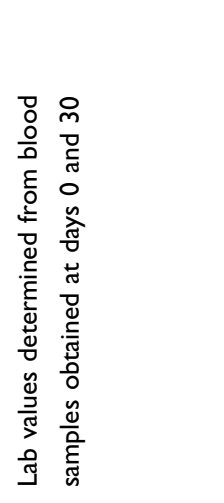 & 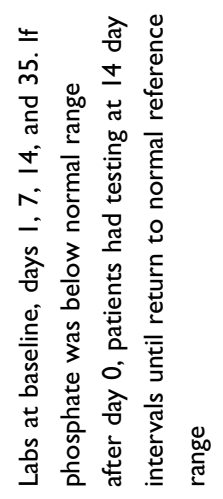 & 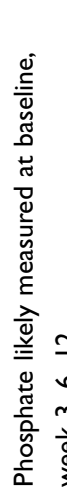 & & 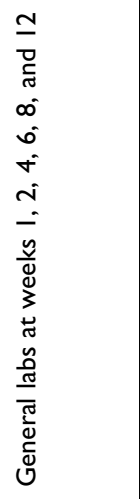 & 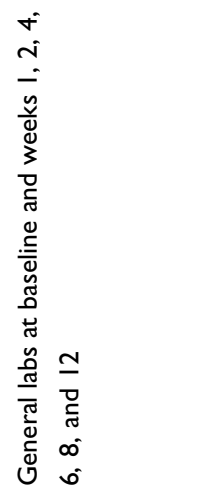 \\
\hline 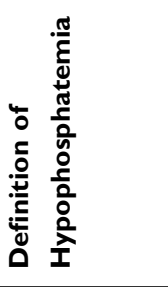 & 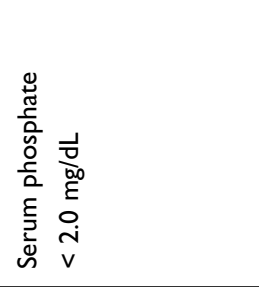 & 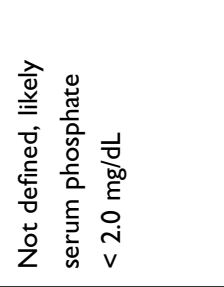 & 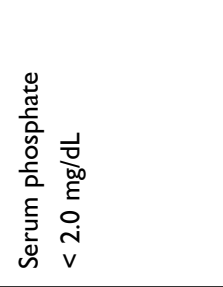 & 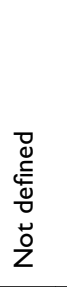 & & 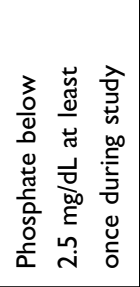 & 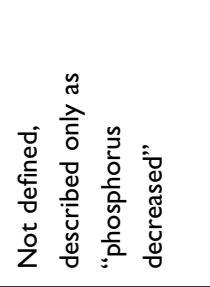 \\
\hline 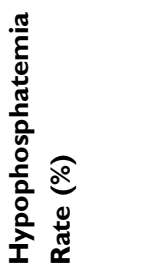 & $\stackrel{\circ}{\stackrel{\circ}{~}}$ & 总 & $\begin{array}{l}\stackrel{\circ}{\infty} \\
\infty \\
\infty\end{array}$ & ○ें & 今̊ & $\frac{\stackrel{\circ}{\alpha}}{\text { d }}$ & $\begin{array}{l}\stackrel{\circ}{\text { ஸे }} \\
\stackrel{\infty}{-}\end{array}$ \\
\hline 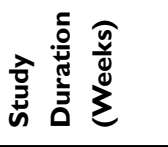 & in & in & in & $\simeq$ & & $\simeq$ & $\simeq$ \\
\hline 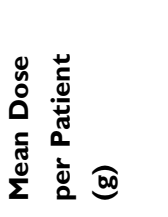 & $\begin{array}{l}\stackrel{n}{\hat{0}} \\
\hat{0}\end{array}$ & \begin{tabular}{l}
\multirow{2}{*}{} \\
o
\end{tabular} & $\bar{o}$ & 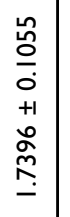 & $\begin{array}{l}\frac{a}{2} \\
\bar{c} \\
+1 \\
+1 \\
0 \\
\stackrel{e}{-}\end{array}$ & 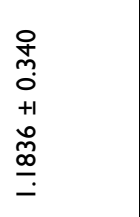 & 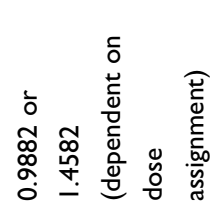 \\
\hline 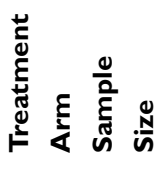 & $\stackrel{m}{m}$ & よ̊ & $\simeq$ & 요 & 요 & aे & $\underline{\underline{a}}$ \\
\hline $\begin{array}{l}\frac{2}{0} \\
\frac{\pi}{5} \\
\frac{0}{2} \\
0 \\
0\end{array}$ & 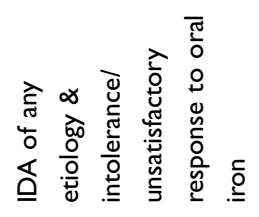 & 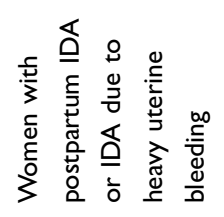 & 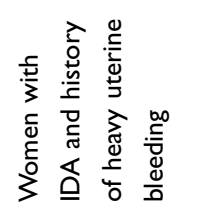 & \multicolumn{2}{|c|}{ 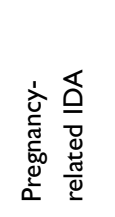 } & 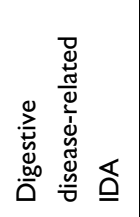 & 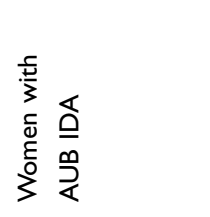 \\
\hline 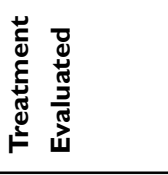 & $\sum_{\cup}$ & $\sum_{U}$ & $\sum_{U}$ & $\sum_{U}$ & 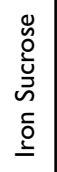 & $\sum_{U}$ & $\sum_{\cup}$ \\
\hline $\begin{array}{l}\text { ते } \\
\text { uे }\end{array}$ & 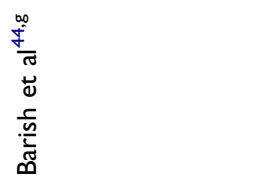 & 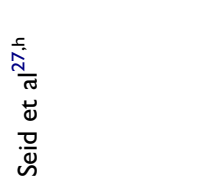 & 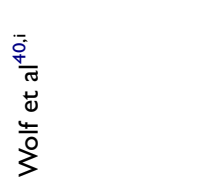 & 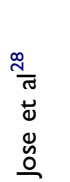 & & 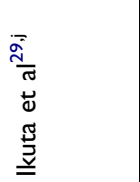 & 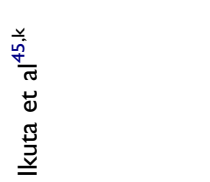 \\
\hline
\end{tabular}




\begin{tabular}{|c|c|c|c|c|c|c|c|c|c|}
\hline \multicolumn{2}{|c|}{ 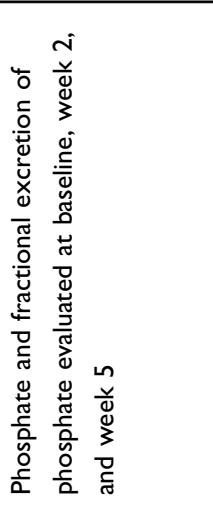 } & 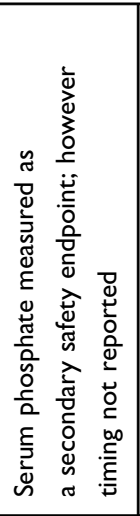 & 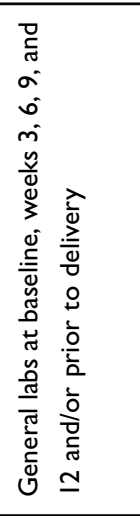 & 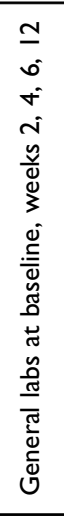 & & 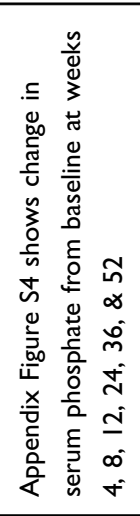 & 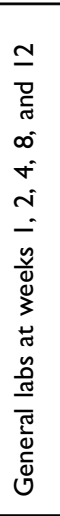 & & \multirow{2}{*}{ 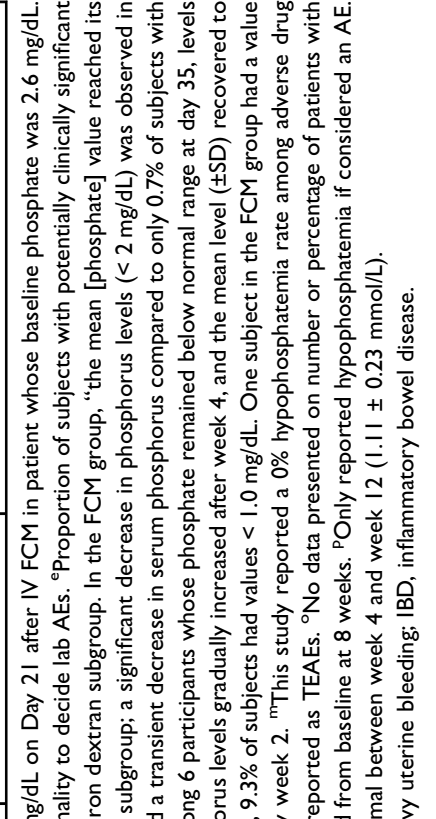 } \\
\hline \multicolumn{2}{|c|}{ 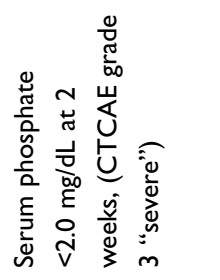 } & 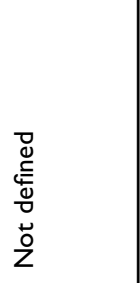 & 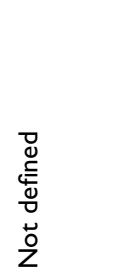 & 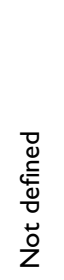 & & 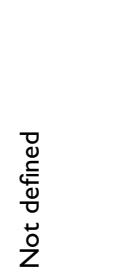 & \multicolumn{2}{|l|}{ 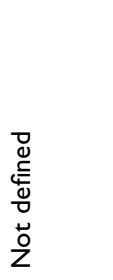 } & \\
\hline $\begin{array}{l}\stackrel{\circ}{\circ} \\
\infty \\
\text { mo }\end{array}$ & ণें & @̊․ㅇ & $\frac{\circ 0}{\infty}$ & 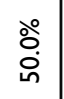 & $\begin{array}{l}\text { ठ̊ํ } \\
\text { ó }\end{array}$ & ठ̊ㅇㅇ & ஸें & oें & $\frac{\pi}{0} \frac{\pi}{0}$ \\
\hline \multicolumn{2}{|l|}{ n } & in & $\simeq$ & $\simeq$ & & กิ & \multicolumn{2}{|l|}{$\simeq$} & 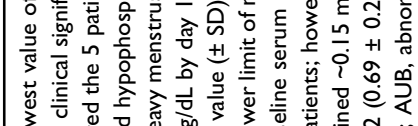 \\
\hline 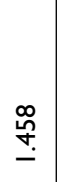 & $\begin{array}{l}\text { d } \\
\text { o }\end{array}$ & $\stackrel{\infty}{\underset{I}{=}}$ & 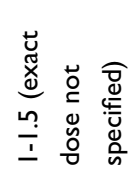 & 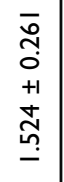 & 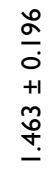 & $\underset{\stackrel{\leftrightarrow}{0}}{\stackrel{\leftrightarrow}{i}}$ & $\begin{array}{l}\bar{\infty} \\
\stackrel{0}{0} \\
0 \\
++1 \\
\stackrel{N}{\stackrel{m}{=}}\end{array}$ & \begin{tabular}{|c|}
$\frac{0}{m}$ \\
0 \\
0 \\
++1 \\
0 \\
$\underline{0}$ \\
- \\
\end{tabular} & 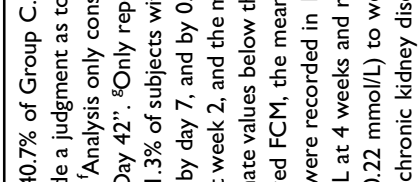 \\
\hline 政 & बू & $\stackrel{\infty}{\stackrel{\infty}{0}}$ & $\cong$ & in & ᄋ & 点 & $\underset{\sim}{\mathbb{J}}$ & స్ & 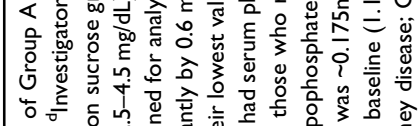 \\
\hline \multicolumn{2}{|c|}{ 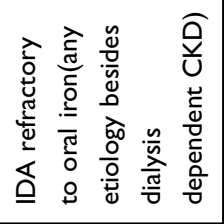 } & 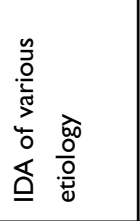 & 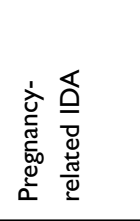 & $\begin{array}{l}\stackrel{1}{0} \\
\stackrel{9}{\varrho}\end{array}$ & & $\begin{array}{l}\text { @̂́ } \\
\text { U U }\end{array}$ & \multicolumn{2}{|l|}{$\begin{array}{l}\text { @̊ } \\
\underline{\underline{O}}\end{array}$} & 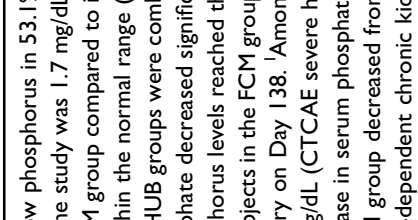 \\
\hline$\sum_{u}$ & 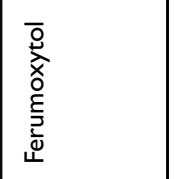 & 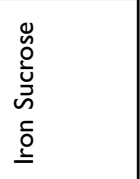 & 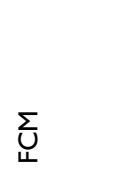 & u & 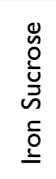 & $\sum_{\cup}$ & & 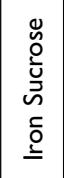 & 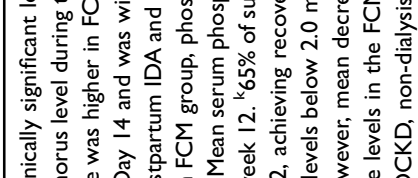 \\
\hline 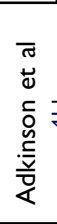 & & 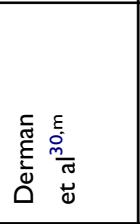 & 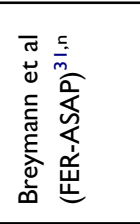 & 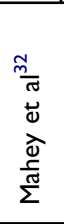 & & 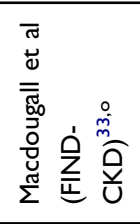 & 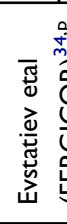 & & 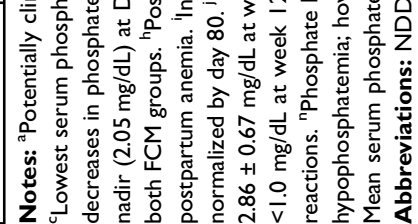 \\
\hline
\end{tabular}




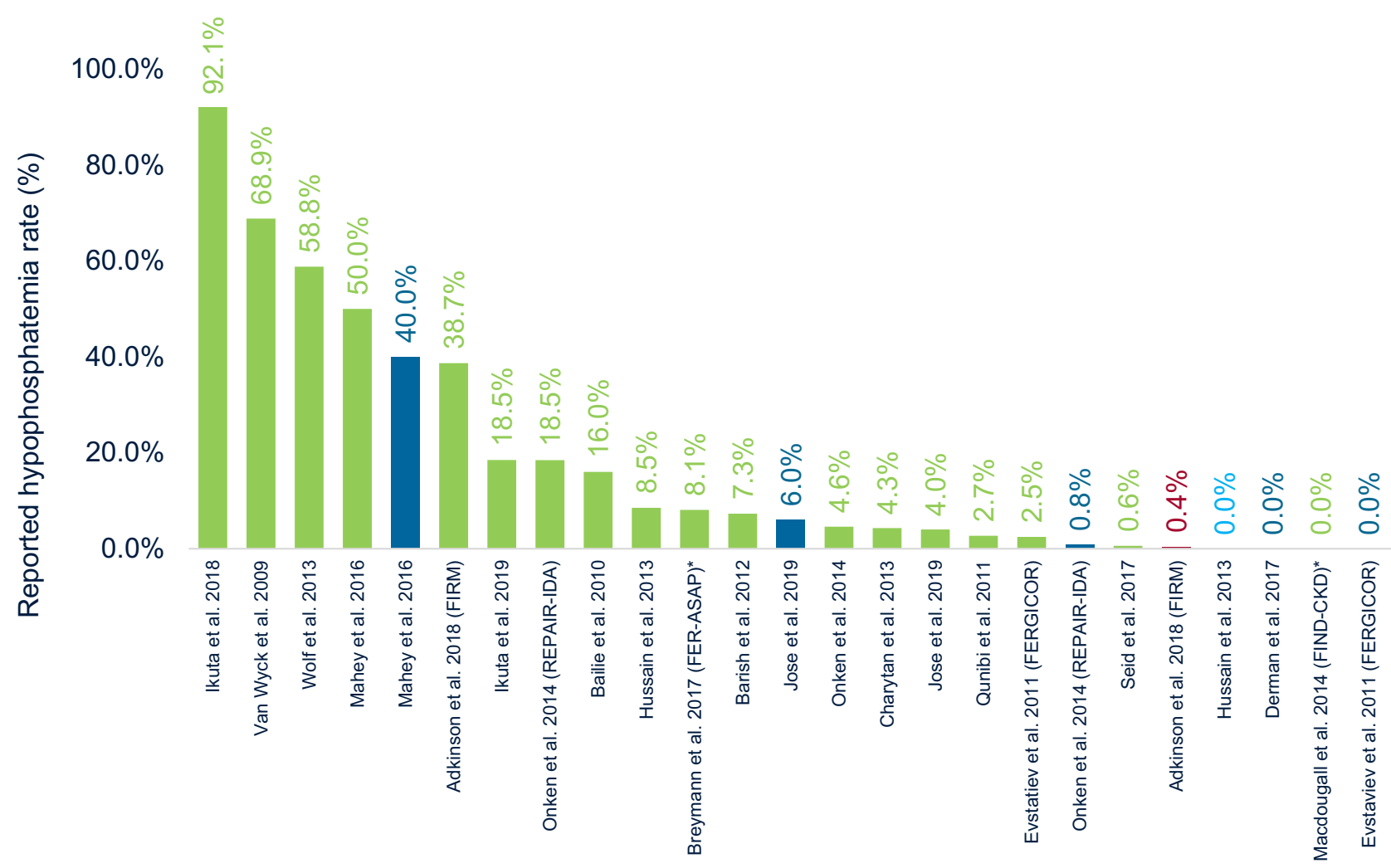

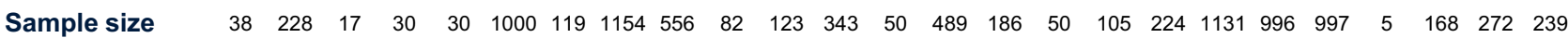

FCM

Iron Sucrose

Ferumoxytol

LMW Iron Dextran

Figure 2 Hypophosphatemia rate (\%) reported per RCT.

Notes: Reported hypophosphatemia rates are given as noted in Table I. Corresponding sample size of study treatment arm is shown below each study title. *Breymann et $\mathrm{al}^{3 \mathrm{I}}$ report $0 \%$ hypophosphatemia treatment-emergent adverse events in patients; however, this study reports serum phosphate levels below the threshold of CTCAE grade 3 hypophosphatemia in $8.1 \%$ of patients. Macdougall et $\mathrm{al}^{33}$ report no "clinically significant hypophosphatemia"; however this study reports phosphate decrease in supplemental figures but without detailed information on number or \% of patients falling below CTCAE threshold.

Abbreviations: RCT, randomized controlled trial; IVI, intravenous iron; FCM, ferric carboxymaltose; LMW, low-molecular-weight.

for iron sucrose, $0.4 \%$ for ferumoxytol, and $0.0 \%$ for LMW iron dextran. All studies either described hypophosphatemia as "asymptomatic" or did not report on other symptoms caused by hypophosphatemia.

Across all studies reviewed, the highest hypophosphatemia rates were observed in patients with IDA due to abnormal uterine bleeding (AUB). Five studies of FCM within AUB patients reported hypophosphatemia ranging from $0.6 \%$ to $68.9 \%$ and a single study of iron sucrose with IDA secondary to AUB reported a rate of $40.0 \%{ }^{16,27,29,32,40}$ The lowest rates of hypophosphatemia were observed in pregnancy-related IDA (range $0.0-6.0 \%)^{27,28,31}$

Reported mean cumulative IVI dosing (MCID) ranged from $750 \mathrm{mg}$ to $2.685 \mathrm{~g}$ across RCTs. Two studies did not explicitly report MCID, giving only ranges. ${ }^{31,45}$ Out of 19
RCTs, 18 reported MCID below 1.75 g. Studies with hypophosphatemia rates greater than $10.0 \%$ reported MCID between $910 \mathrm{mg}$ and $1.568 \mathrm{~g}$. Cumulative dosing across studies appeared largely determined by FDAapproved labeling. Studies did not report a clear relationship between cumulative IVI dose and treatment-emergent hypophosphatemia. Figure 3 plots hypophosphatemia rates against MCID by IVI treatment within each RCT study.

\section{Level II Evidence}

Ten observational, retrospective, or post hoc studies were identified as a supplemental data source to RCT articles (Figure 4). ${ }^{17,35-38,42,46-49}$ All ten studies investigated FCM; two also reported on iron sucrose treatment arms. ${ }^{17,38}$ Level II studies reported hypophosphatemia rates ranging $0.0-82.0 \%$ with FCM and $0.0-22.0 \%$ with iron sucrose. 


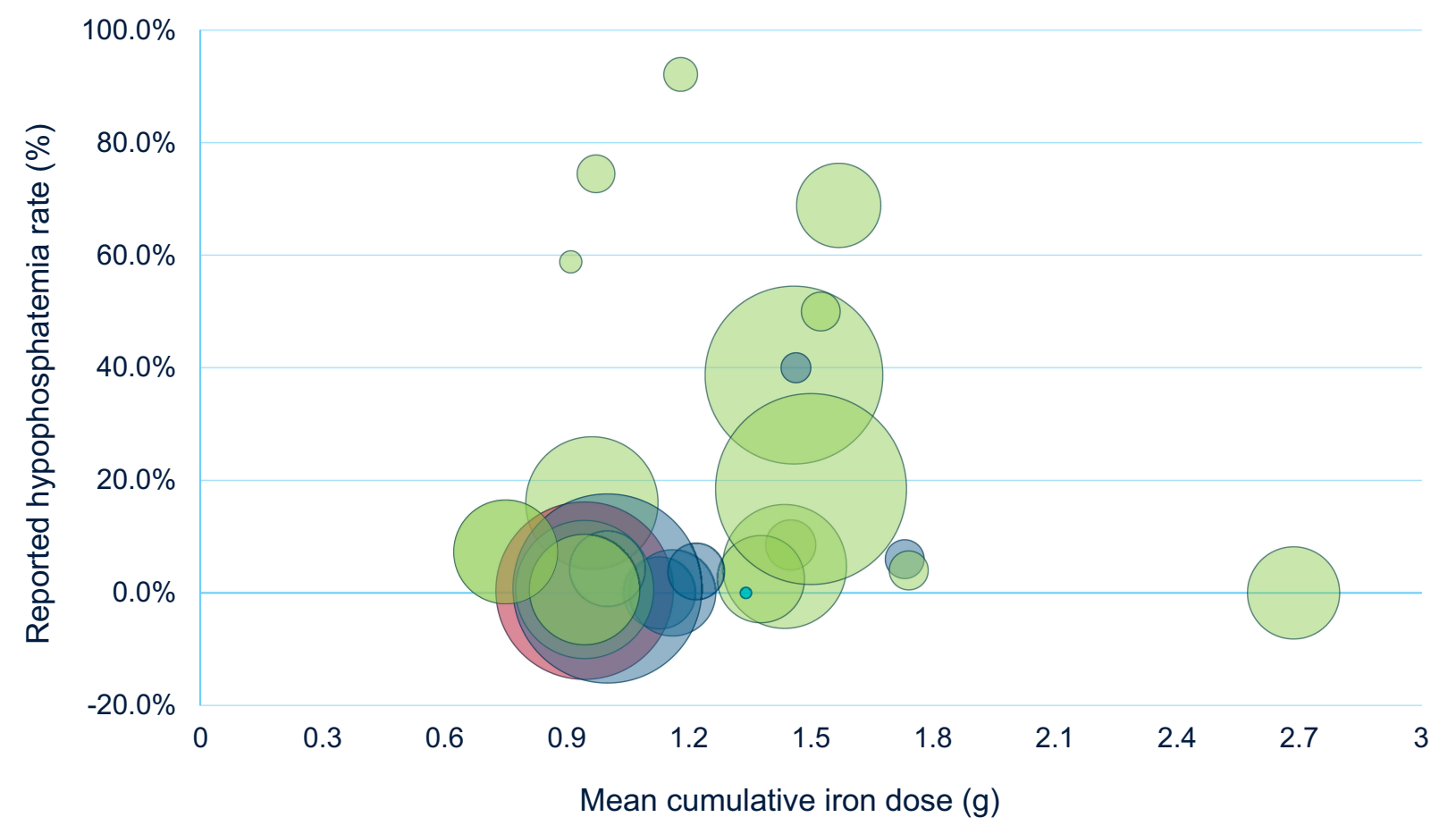

FCM

Iron Sucrose

Ferumoxytol

LMW Iron Dextran

Figure 3 Hypophosphatemia rate (\%) reported per RCT vs mean cumulative iron dose (g) by IVI treatment within each RCT.

Notes: Reported hypophosphatemia rates are given as noted in Table I. Bubble size shown is proportional to the sample size of treatment arm within the study; cumulative iron doses shown are derived from study summary statistics of cumulative dosing.

Abbreviations: RCT, randomized controlled trial; IVI, intravenous iron; FCM, ferric carboxymaltose; LMW, low-molecular-weight.

Many Level II studies lacked detailed summary statistics for the analyzed sample, including 4 of ten that did not explicitly report dosing of administered IVIs. ${ }^{17,36,38,47}$ Seven of ten observational studies described hypophosphatemia as "asymptomatic" or "transient" due to spontaneous resolution after intervention cessation and no need for further interventions. ${ }^{35-38,47-49}$ A retrospective review of patient medical records by Hardy et al (2015) reported that some FCM-treated patients experienced persistent fatigue despite anemia correction as a clinical consequence of hypophosphatemia. ${ }^{17}$ Two other studies proposed further investigation of potential clinical consequences of hypophosphatemia such as bone disease development, osteomalacia and other long-term outcomes associated with repeat IVI treatments. ${ }^{42,46}$

\section{Level III Evidence}

Eleven case reports evaluating treatment-emergent hypophosphatemia after IVI administration in patients receiving FCM were identified as Level III evidence (Appendix B) ${ }^{18,49-58}$ Five cases reported treatment-emergent hypophosphatemia with severe muscle weakness and fatigue..$^{50,52,54,57,58}$ Four reported hypophosphatemic osteomalacia. ${ }^{18,53,55,56}$ The remaining two case reports described asymptomatic hypophosphatemia. ${ }^{49,51}$

Severe weakness and fatigue were reported in 5 patients who received two doses of $500 \mathrm{mg}$ FCM separated by a 1-week interval. ${ }^{50,52,54,57,58}$ IDA etiology varied among these cases: 2 patients presented with AUB, ${ }^{50,57} 1$ each with IDA secondary to IBD $^{58}$ and renal transplant, ${ }^{54}$ and 1 with severe iron deficiency without anemia. ${ }^{52}$ Patients reported either no improvement of fatigue or complained about worsening weakness after treatment with FCM. In addition to generalized weakness, headache and respiratory distress were also reported in 1 patient each. ${ }^{57,58}$

Hypophosphatemic osteomalacia was detailed in 4 cases of patients receiving repeated FCM doses of either 750 or 1000 mg. ${ }^{18,53,55,56}$ The duration of patients' repeated FCM therapy regimen ranged from 8 months to 15 years. All patients had experienced severe bone pain and recurrent fractures that persisted for months to years.

Clinical sequelae due to IVI-induced hypophosphatemia were eventually reversed in all but 1 case. ${ }^{55}$ In patients with severe weakness and fatigue, symptoms resolved on 


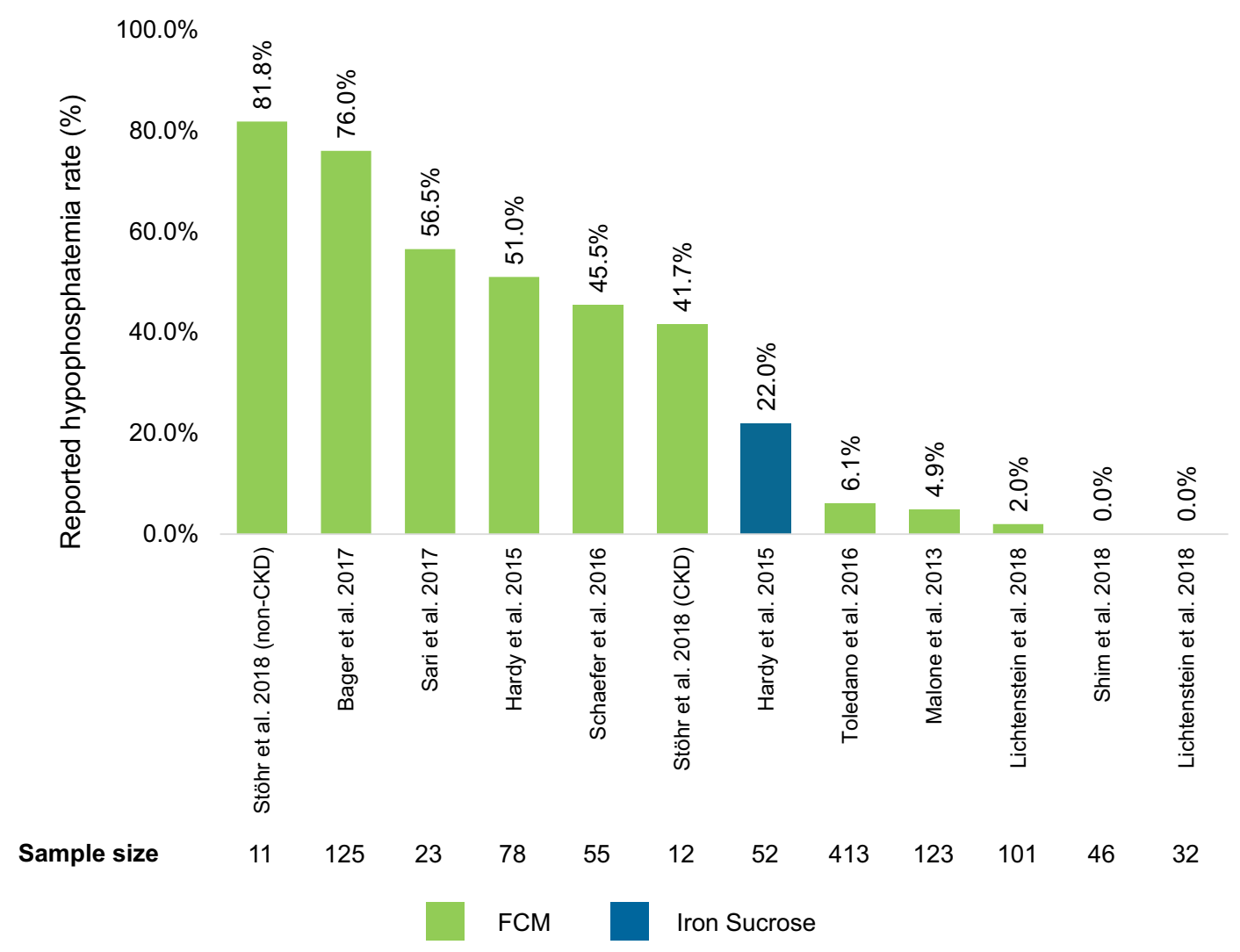

Figure 4 Hypophosphatemia rate (\%) reported by individual non-RCT publication.

Notes: Reported hypophosphatemia rates given as noted in Table I. The rate of hypophosphatemia reported by study treatment arm was taken using definitions for hypophosphatemia included within each study.

Abbreviation: Non-RCT, non-randomized controlled trial.

normalization of serum phosphate (range 2 weeks to 2 months). In patients with hypophosphatemic osteomalacia, full resolution of osteomalacia took 5 to 12 months. In 1 case, hypophosphatemic osteomalacia resolved, but severe bone deformities were reported, which were considered likely to be permanent. ${ }^{55}$ A search conducted in August 2019 identified 5 additional articles published since the inception of this manuscript, but these were not included in the detailed analysis due to publication after the original search was performed.

\section{Discussion}

Phosphate plays an essential role in the management of metabolism, enzymatic function, bone mineralization, and cellular structure. ${ }^{59}$ Depression of phosphate homeostasis may therefore lead to serious short- and long-term clinical consequences including fatigue, osteomalacia, rhabdomyolysis, and even death depending on its severity and duration. Although the precise mechanism by which IVIs may cause hypophosphatemia remains incompletely understood, recent literature suggests that certain parenteral iron preparations may increase the urinary fractional excretion of phosphate via modulation of the intracellular metabolism of fibroblast growth factor 23 (FGF23). ${ }^{40,60,61}$ Despite the suggestion of older studies that hypophosphatemia may be due simply to higher intracellular phosphate uptake coincident with increased erythropoiesis, recent studies have demonstrated no significant correlation between erythropoiesis (as measured by increase in $\mathrm{Hgb}$ ) or total iron dose and rates of hypophosphatemia. ${ }^{16,60}$

Although hypophosphatemia has been recognized as a potential adverse event associated with IVIs, only a small proportion of studies indexed in this literature review reported hypophosphatemia rates or changes in serum phosphate levels. Out of 62 US-based or global RCTs investigating US-marketed IVIs for IDA treatment included in this study, only 19 presented data on rates of treatment-emergent hypophosphatemia or serum phosphate changes. When reported, rates of treatment-emergent hypophosphatemia within the literature varied extensively across RCTs, observational, retrospective, and post hoc studies. Differences in study designs and data analysis may account 
for this inconsistency. Within the literature reviewed, our analysis found (1) inconsistent inclusion of serum phosphate and hypophosphatemia as end-points of interest in studies of IVIs within IDA, (2) a lack of a standard approach and timeline for measurement of phosphate levels, and (3) significant variability in the reporting, definitions and follow-up of any hypophosphatemia observed. These findings indicate that there is a clear need for additional rigorous and standardized research into hypophosphatemia as a clinical consequence of IVI administration.

Furthermore, the majority of studies reviewed did not explicitly report serum phosphate measurement methodology or definitions of hypophosphatemia used for analysis. Although serum phosphate may have been analyzed alongside other hematological parameters recorded within studies, only a minority of publications actively described phosphate measurement when reporting study methodologies. Importantly, timepoints of serum phosphate measurement were not explicitly reported in most Level I and II studies. Within the Level II cohort in particular, timepoints of serum phosphate measurement were neither reported nor consistent among patients, even within the same study. The reported rates of hypophosphatemia may therefore vary significantly as a result of the timing of post-treatment measurement of serum phosphate levels in these studies.

In addition to inconsistent serum phosphate measurement methodologies, reported definitions of hypophosphatemia were either absent or highly variable across studies. Within the minority of studies that did report hypophosphatemia definitions, serum phosphate reference ranges and other qualifying criteria differed significantly. Although CTCAE guidelines for the classification of hypophosphatemia exist, only a few publications explicitly stated the use of this reference range in designating hypophosphatemia within study populations. ${ }^{43}$ Moreover, even if the CTCAE suggested reference ranges were used, an investigator's choice of units may have contributed to variations in reported rates of hypophosphatemia. CTCAE guidelines suggest the upper limit of Grade 3 ("severe") hypophosphatemia to be either $0.6 \mathrm{mmol} / \mathrm{L}$ or $2.0 \mathrm{mg} / \mathrm{dL}$; however, these figures are not precisely equal, with $0.6 \mathrm{mmol} / \mathrm{L}$ actually corresponding to slightly less than $1.9 \mathrm{mg} / \mathrm{dL}$ (requiring a lower serum phosphate level to be considered hypophosphatemic compared to $2.0 \mathrm{mg} / \mathrm{dL}$ ). Therefore, it is also plausible that the use of differing reference ranges and classification criteria for hypophosphatemia could have contributed to the inconsistent reporting.
Our review also identified several studies in which hypophosphatemia rates appeared to be reported inconsistently across trial arms or appeared to diverge from reported changes in serum phosphate. For example, Barish et al (2012) only reported rates of hypophosphatemia in 1 study arm (multi-dose patients) despite citing statistically significant changes in phosphorus levels within all study subgroups. ${ }^{44}$ Seid et al (2017) reported a cumulative FCM hypophosphatemia rate of $0.6 \%$ despite reporting a statistically significant transient phosphate decrease in $9.0 \%$ of all patients dosed with FCM versus $0.0 \%$ of patients given standard medical care (no explicit definition of hypophosphatemia was mentioned). A subgroup analysis within this study additionally cited a transient decrease in serum phosphorus in $21.3 \%$ of AUB and $0.7 \%$ of pregnancy-related IDA patients, respectively. Ikuta et al (2019) reported hypophosphatemia in $18.5 \%$ of patients despite acknowledging that $65.0 \%$ of subjects dosed with FCM had serum phosphorus levels "below the lower limit of normal" at week $1 .{ }^{45}$ Until definitions and consistent measurement of hypophosphatemia as well as detailed reporting of serum phosphate are systematically included in all trials and clinical practice, it may continue to be difficult to fully understand the severity and magnitude of treatmentemergent hypophosphatemia.

The studies analyzed within this review may have underestimated the occurrence of hypophosphatemia and its hypothesized clinical consequences due to the short duration of dosing regimens and follow-up evaluation used within study protocols. Only 1 trial among the Level I evidence cohort, FIND-CKD, reported data on hypophosphatemia rates in patients dosed for over 12 weeks. ${ }^{33}$ Consequently, the long-term effects of hypophosphatemia or repeat IVI dosing may not have been taken into consideration in most RCTs.

Although trials reporting hypophosphatemia noted the condition to be "asymptomatic" or "transient" in patients, it is important to note that certain symptoms of hypophosphatemia-namely fatigue-may present identically to those of IDA and thus may not have been recognized by trial investigators as treatment-emergent adverse events. In contrast to the "asymptomatic" hypophosphatemia reported in trials, case reports demonstrate that IVI-induced hypophosphatemia may be a serious clinical consideration. Patient cases in the literature detail short-term consequences of severe muscle weakness and fatigue and long-term concerns of fractures and bone deformities due to hypophosphatemic osteomalacia in patients undergoing repeat IVI 
dosing. Although hypophosphatemia was reversed in all cases reviewed, patients were forced to discontinue IVI therapy and required weeks or months-long courses of phosphate and calcitriol supplementation. These patients additionally needed to undergo long-term evaluation by their care teams to prevent further complications.

Similarly, although hypophosphatemia was reported as "transient" in many of the Level I and II studies, evidence of adverse events secondary to chronic hypophosphatemia in case reports of patients undergoing repeat dosing with FCM indicates that there may be long-term health effects not captured in current trial designs. For instance, studies have indicated that nadir serum phosphate in patients given certain IVIs may occur approximately 2 to 3 weeks after IVI administration; however, research has shown that a significant percentage of patients continue to manifest severe hypophosphatemia at 5 weeks. $^{62}$ In addition, although serum phosphate typically recovered to baseline by the end of the study period for many patients described within trials, there may be many patients in the real-world setting who regularly receive repeat dosing of IVIs as often as once every 4 weeks. In these patients, as with those detailed in the case reports, there may be a potential "stacking" effect of hypophosphatemia - with patients unable to recover serum phosphate to baseline by the time of their next infusion. Although these populations have not been systematically studied within the literature, they may be at risk of chronic hypophosphatemia and thus susceptible to the serious consequences of persistently depressed serum phosphate levels such as hypophosphatemic osteomalacia.

Findings of this literature review suggest that patients with normal renal function and higher iron dose per $\mathrm{kg}$ are at highest risk of developing hypophosphatemia. ${ }^{60,62}$ These findings are supported by a post hoc analysis of the FIRM trial population conducted by Wolf et al (2018) demonstrating an increased risk of hypophosphatemia in patients without CKD. ${ }^{62}$ Another clinical risk factor for the development of hypophosphatemia reported in the literature was the presence of IDA due to AUB; CKD and pregnancy-related IDA appeared to be associated with lower risk. ${ }^{16,62}$ The AUB population may be a particularly at-risk subgroup of patients who could potentially benefit from frequent and timely evaluations for hypophosphatemia symptoms.

Lastly, among all IVI therapies investigated within this review, FCM displayed the highest rates of hypophosphatemia. This finding did not appear to differ for any subgroup of IDA patients examined. Recent head-to-head studies of treatment-emergent hypophosphatemia in IDA patients have indicated that of all risk factors for the development of hypophosphatemia, the use of FCM as opposed to another treatment was the most predictive. ${ }^{62}$ There did not appear to be a class-wide correlation between hypophosphatemia and iron dose administered with IVI therapies. Furthermore, the efficacy observed in IVI patients did not appear to be correlated with hypophosphatemia development.

\section{Limitations}

This SLR had several limitations owing to the inherent nature of literature review methodology, restricted structure of our search, and the heterogeneity of available data sources. This study was focused on US-marketed IVIs and thus products not marketed in the US at the time of the literature review were excluded from our analysis. Since the time of our literature search, iron isomaltoside has been approved by the US Food and Drug Administration (as ferric derisomaltose). A newly published article by Wolf et al (2020) has reported rates of hypophosphatemia from 2 randomized controlled trials comparing iron isomaltoside and ferric carboxymaltose $(7.9-8.1 \%$ and $73.7-75.0 \%$, respectively). ${ }^{63}$ Patient populations' IDA etiology and baseline anemia status differed significantly across the included studies and case reports. The observed variability in IVI dosing regimens, timing of phosphate measurement, serum phosphate reference ranges and definitions of hypophosphatemia may additionally confound reported hypophosphatemia rates. This heterogeneity of available sources precluded a robust quantitative and statistical analysis of hypophosphatemia and its associated clinical sequelae. Findings of this study should thus be interpreted with these caveats in mind. Despite these limitations, this study's findings suggest that there may be value in additional scientific and realworld studies on this topic, designed to control for underlying patient conditions and etiologies which consistently define, measure and report hypophosphatemia rates.

\section{Conclusion}

In summary, our analysis suggests that there is a significant need for further research to elucidate the mechanism of IVI-induced hypophosphatemia as well as for more consistent inclusion, measurement, and reporting of serum phosphate levels as an endpoint in clinical trials. Moving forward, research and guidelines should aim to standardize the definitions of hypophosphatemia and suggest specific timing for serum phosphate measurement within clinical trials as well as real-world studies. Current CTCAE guidelines for hypophosphatemia may be a good reference point 
for future studies. Given the potential clinical impact of IVI-induced hypophosphatemia, particularly with repeated dosing of certain IVIs, further research, will also be needed to assess the effect of various dosing regimens on long-term serum phosphate levels.

Current studies may be significantly underestimating rates of treatment-emergent hypophosphatemia due to inconsistent and infrequent measurement of serum phosphate, inconsistent definitions of hypophosphatemia, and similarity between the symptoms of hypophosphatemia and anemia. Across all studies, the highest rates of hypophosphatemia were seen with FCM. All case reports profiled severe weakness and fatigue or hypophosphatemic osteomalacia in FCM patients who developed treatmentinduced hypophosphatemia.

Hypophosphatemia may not only impact patients' clinical outcomes and quality of life in the short- and long term, but may also create a burden on the healthcare system due to the need for additional medications and close monitoring of patients. Until the true prevalence and clinical impact of IVIinduced hypophosphatemia is more fully characterized and quantified within the literature, findings of this SLR suggest that physicians and researchers actively consider the possibility of hypophosphatemia in all patients receiving IVIs, particularly in those receiving therapies such as FCM, which has been associated with elevated rates of treatmentemergent hypophosphatemia in various clinical studies. Given that the symptoms of hypophosphatemia may appear almost identical to those of IDA, healthcare professionals should remain aware of the possibility of hypophosphatemia in their IDA patients, and carefully and consistently monitor pre- and post-treatment serum phosphate levels in all patients receiving IVIs.

Of note, during the finalization of this manuscript, the US FDA revised the prescribing information for Injectafer (ferric carboxymaltose), adding a warning that severe symptomatic episodes of hypophosphatemia requiring clinical intervention have occurred following Injectafer administrations and recommending serum phosphate monitoring in patients at risk for hypophosphatemia who require a repeat course of treatment. These labeling changes highlight both the relevance of the conclusions of this analysis and the need for heightened awareness of this phenomenon.

\section{Author Contributions}

The authors were responsible for all content and editorial decisions related to the development of this manuscript. All authors contributed to data analysis, drafting or revising the article, gave final approval of the version to be published, and agree to be accountable for all aspects of the work.

\section{Funding}

This study was sponsored by AMAG Pharmaceuticals, Inc. Editorial support in the preparation of this manuscript was provided by Trinity LifeSciences, funded by AMAG Pharmaceuticals, Inc.

\section{Disclosure}

MZ Lim-Watson was an employee of AMAG Pharmaceuticals, Inc at the time the study was conducted. A. Bajic-Lucas, NV Dahl, and WE Strauss are full-time employees of AMAG Pharmaceuticals, Inc. and hold equity in AMAG Pharmaceuticals, Inc. MA Libre, SS Karkare, and $\mathrm{N}$ Hadker are employees of Trinity LifeSciences, which has conducted research for AMAG Pharmaceuticals, Inc. JA Glaspy is a consultant for AMAG Pharmaceuticals, Inc. The authors report no other conflicts of interest in this work.

\section{References}

1. Lopez A, Cacoub P, Macdougall IC, Peyrin-Biroulet L. Iron deficiency anaemia. Lancet. 2016;387(10021):907-916. doi:10.1016/ S0140-6736(15)60865-0

2. Camaschella C. Iron-deficiency anemia. Longo DL, ed. $N$ Engl $J$ Med. 2015;372(19):1832-1843. doi:10.1056/NEJMra1401038

3. Miller JL. Iron deficiency anemia: a common and curable disease. Cold Spring Harb Perspect Med. 2013;3(7):a011866-a011866. doi:10.1101/cshperspect.a011866

4. Vos T, Abajobir AA, Abate KH, et al. Global, regional, and national incidence, prevalence, and years lived with disability for 328 diseases and injuries for 195 countries, 1990-2016: a systematic analysis for the Global Burden of Disease Study 2016. Lancet. 2017;390 (10100):1211-1259. doi:10.1016/S0140-6736(17)32154-2

5. Auerbach M, Adamson JW. How we diagnose and treat iron deficiency anemia. Am J Hematol. 2016;91(1):31-38. doi:10.1002/ajh.24201

6. Tolkien Z, Stecher L, Mander AP, Pereira DIA, Powell JJ. Ferrous sulfate supplementation causes significant gastrointestinal side-effects in adults: a systematic review and meta-analysis. Strnad Ped. PLoS One. 2015;10(2):e0117383. doi:10.1371/journal.pone.0117383

7. Bregman DB, Morris D, Koch TA, He A, Goodnough LT. Hepcidin levels predict nonresponsiveness to oral iron therapy in patients with iron deficiency anemia. Am J Hematol. 2013;88(2):97-101. doi:10.1002/ ajh.23354

8. Reinisch W, Chowers Y, Danese S, et al. The management of iron deficiency in inflammatory bowel disease - an online tool developed by the RAND/UCLA appropriateness method. Aliment Pharmacol Ther. 2013;38(9):1109-1118. doi:10.1111/apt.12493

9. Onken JE, Bregman DB, Harrington RA, et al. A multicenter, randomized, active-controlled study to investigate the efficacy and safety of intravenous ferric carboxymaltose in patients with iron deficiency anemia. Transfusion. 2013;54(2):n/a-n/a. doi:10.1111/trf.12289

10. Vadhan-Raj S, Strauss W, Ford D, et al. Efficacy and safety of IV ferumoxytol for adults with iron deficiency anemia previously unresponsive to or unable to tolerate oral iron. Am J Hematol. 2014;89 (1):7-12. doi:10.1002/ajh.23582 
11. Auerbach M, Ballard H. Clinical use of intravenous iron: administration, efficacy, and safety. Hematol. 2010;2010(1):338-347. doi:10.11 82/asheducation-2010.1.338

12. Ehlken B, Nathell L, Gohlke A, Bocuk D, Toussi M, Wohlfeil S. Evaluation of the reported rates of severe hypersensitivity reactions associated with ferric carboxymaltose and iron (III) isomaltoside 1000 in Europe based on data from EudraVigilance and VigiBase ${ }^{\mathrm{TM}}$ between 2014 and 2017. Drug Saf. 2019;42(3):463-471. doi:10.1007/ s40264-018-0769-5

13. Wang C, Graham DJ, Kane RC, et al. Comparative risk of anaphylactic reactions associated with intravenous iron products. JAMA. 2015;314(19):2062. doi:10.1001/jama.2015.15572

14. European Medicines Agency. New recommendations to manage risk of allergic reactions with intravenous iron-containing medicines the European Medicines Agency's Committee for Medicinal Products for Human Use; 2013. Available from: www.ema.europa.eu. Accessed June 26, 2019.

15. Rampton D, Folkersen J, Fishbane S, et al. Hypersensitivity reactions to intravenous iron: guidance for risk minimization and management. Haematologica. 2014;99(11):1671-1676. doi:10.3324/haematol.201 4.111492

16. Van Wyck DB, Mangione A, Morrison J, Hadley PE, Jehle JA, Goodnough LT. Blood management: large-dose intravenous ferric carboxymaltose injection for iron deficiency anemia in heavy uterine bleeding: a randomized, controlled trial. Transfusion. 2009;49 (12):2719-2728. doi:10.1111/j.1537-2995.2009.02327.x

17. Hardy S, Vandemergel X. Intravenous iron administration and hypophosphatemia in clinical practice. Int $J$ Rheumatol. 2015;2015:1-6. doi: $10.1155 / 2015 / 468675$

18. Bartko J, Roschger P, Zandieh S, Brehm A, Zwerina J, Klaushofer K. Hypophosphatemia, severe bone pain, gait disturbance, and fatigue fractures after iron substitution in inflammatory bowel disease: a case report. J Bone Miner Res. 2018;33(3):534-539. doi:10.1002/jbmr. 3319

19. Liamis G, Milionis HJ, Elisaf M. Medication-induced hypophosphatemia: a review. QJM. 2010;103(7):449-459. doi:10.1093/qjmed/hcq 039

20. Brunelli SM, Goldfarb S. Hypophosphatemia: clinical consequences and management. J Am Soc Nephrol. 2007;18(7):1999-2003. doi:10. 1681/ASN.2007020143

21. Imel EA, Econs MJ. Approach to the hypophosphatemic patient. J Clin Endocrinol Metab. 2012;97(3):696-706. doi:10.1210/jc.20111319

22. Avni T, Bieber A, Grossman A, Green H, Leibovici L, Gafter-Gvili A. The safety of intravenous iron preparations. Mayo Clin Proc. 2015;90(1):12-23. doi:10.1016/j.mayocp.2014.10.007

23. Qunibi WY, Martinez C, Smith M, Benjamin J, Mangione A, Roger SD. A randomized controlled trial comparing intravenous ferric carboxymaltose with oral iron for treatment of iron deficiency anaemia of non-dialysis-dependent chronic kidney disease patients. Nephrol Dial Transplant. 2011;26(5):1599-1607. doi:10.1093/ndt/ gfq613

24. Charytan C, Bernardo MV, Koch TA, Butcher A, Morris D, Bregman DB. Intravenous ferric carboxymaltose versus standard medical care in the treatment of iron deficiency anemia in patients with chronic kidney disease: a randomized, active-controlled, multi-center study. Nephrol Dial Transplant. 2013;28(4):953-964. doi:10.1093/ndt/gfs528

25. Bailie GR, Mason NA, Valaoras TG. Safety and tolerability of intravenous ferric carboxymaltose in patients with iron deficiency anemia. Hemodial Int. 2010;14(1):47-54. doi:10.1111/j.1542-4758. 2009.00409.x

26. Onken JE, Bregman DB, Harrington RA, et al. Ferric carboxymaltose in patients with iron-deficiency anemia and impaired renal function: the REPAIR-IDA trial. Nephrol Dial Transplant. 2014;29(4):83 3-842. doi:10.1093/ndt/gft251
27. Seid MH, Butcher AD, Chatwani A. Ferric carboxymaltose as treatment in women with iron-deficiency anemia. Anemia. 2017;2017:1-9. doi:10.1155/2017/9642027

28. Jose A, Mahey R, Sharma JB, et al. Comparison of ferric Carboxymaltose and iron sucrose complex for treatment of iron deficiency anemia in pregnancy-randomised controlled trial. BMC Pregnancy Childbirth. 2019;19(1):54. doi:10.1186/s12884-0192200-3

29. Ikuta K, Ito H, Takahashi K, Masaki S, Terauchi M, Suzuki Y. Safety and efficacy of intravenous ferric carboxymaltose in Japanese patients with iron-deficiency anemia caused by digestive diseases: an open-label, single-arm study. Int J Hematol. 2019;109(1):50-58. doi:10.1007/s12185-018-2529-9

30. Derman R, Roman E, Modiano MR, Achebe MM, Thomsen LL, Auerbach M. A randomized trial of iron isomaltoside versus iron sucrose in patients with iron deficiency anemia. Am J Hematol. 2017;92(3):286-291. doi:10.1002/ajh.24633

31. Breymann C, Milman N, Mezzacasa A, Bernard R, Dudenhausen J. FER-ASAP investigators. Ferric carboxymaltose vs. oral iron in the treatment of pregnant women with iron deficiency anemia: an international, open-label, randomized controlled trial (FER-ASAP). $J$ Perinat Med. 2017;45(4):443-453. doi:10.1515/jpm-2016-0050

32. Mahey R, Kriplani A, Mogili KD, Bhatla N, Kachhawa G, Saxena R. Randomized controlled trial comparing ferric carboxymaltose and iron sucrose for treatment of iron deficiency anemia due to abnormal uterine bleeding. Int J Gynecol Obstet. 2016;133(1):43-48. doi:10.10 16/j.ijgo.2015.09.007

33. Macdougall IC, Bock AH, Carrera F, et al. FIND-CKD: a randomized trial of intravenous ferric carboxymaltose versus oral iron in patients with chronic kidney disease and iron deficiency anaemia. Nephrol Dial Transplant. 2014;29(11):2075-2084. doi:10.1093/ndt/gfu201

34. Evstatiev R, Marteau P, Iqbal T, et al. FERGIcor, a randomized controlled trial on ferric carboxymaltose for iron deficiency anemia in inflammatory bowel disease. Gastroenterol. 2011;141(3):846-853. e2. doi:10.1053/j.gastro.2011.06.005

35. Shim J-Y, Kim MY, Kim YJ, et al. Efficacy and safety of ferric carboxymaltose versus ferrous sulfate for iron deficiency anemia during pregnancy: subgroup analysis of Korean women. BMC Pregnancy Childbirth. 2018;18(1):349. doi:10.1186/s12884-0181817-y

36. Toledano A, Luporsi E, Morere JF, et al. Clinical use of ferric carboxymaltose in patients with solid tumours or haematological malignancies in France. Support Care Cancer. 2016;24(1):67-75. doi:10.1007/s00520-015-2728-3

37. Malone M, Barish C, He A, Bregman D. Comparative review of the safety and efficacy of ferric carboxymaltose versus standard medical care for the treatment of iron deficiency anemia in bariatric and gastric surgery patients. Obes Surg. 2013;23(9):1413-1420. doi:10.10 07/s11695-013-0939-6

38. Lichtenstein GR, Onken JE. Improved hemoglobin response with ferric carboxymaltose in patients with gastrointestinal-related iron-deficiency anemia versus oral iron. Dig Dis Sci. 2018;63 (11):3009-3019. doi:10.1007/s10620-018-5204-3

39. Hussain I, Bhoyroo J, Butcher A, Koch TA, He A, Bregman DB. Direct comparison of the safety and efficacy of ferric carboxymaltose versus iron dextran in patients with iron deficiency anemia. Anemia. 2013;2013:169107. doi:10.1155/2013/169107

40. Wolf M, Koch TA, Bregman DB. Effects of iron deficiency anemia and its treatment on fibroblast growth factor 23 and phosphate homeostasis in women. J Bone Miner Res. 2013;28(8):1793-1803. doi:10. 1002/jbmr. 1923

41. Adkinson NF, Strauss WE, Macdougall IC, et al. Comparative safety of intravenous ferumoxytol versus ferric carboxymaltose in iron deficiency anemia: a randomized trial. Am J Hematol. 2018;93 (5):683-690. doi:10.1002/ajh.25060 
42. Prats M, Font R, García C, Cabré C, Jariod M, Vea AM. Effect of ferric carboxymaltose on serum phosphate and C-terminal FGF23 levels in non-dialysis chronic kidney disease patients: post-hoc analysis of a prospective study. BMC Nephrol. 2013;14(1):167. doi:10.11 86/1471-2369-14-167

43. Cancer Institute N. Common Terminology Criteria for Adverse Events (CTCAE) Version 4.0; 2009. Available from: http://www. meddramsso.com. Accessed June 19, 2019.

44. Barish CF, Koch T, Butcher A, Morris D, Bregman DB. Safety and efficacy of intravenous ferric carboxymaltose $(750 \mathrm{mg})$ in the treatment of iron deficiency anemia: two randomized, controlled trials. Anemia. 2012;2012:1-9. doi:10.1155/2012/172104

45. Ikuta K, Hanashi H, Hirai K, et al. Comparison of efficacy and safety between intravenous ferric carboxymaltose and saccharated ferric oxide in Japanese patients with iron-deficiency anemia due to hypermenorrhea: a multi-center, randomized, open-label noninferiority study. Int J Hematol. 2019;109(1):41-49. doi:10.1007/s12185-018-2501-8

46. Schaefer B, Würtinger P, Finkenstedt A, et al. Choice of high-dose intravenous iron preparation determines hypophosphatemia risk Pantopoulos K ed. PLoS One. 2016;11(12):e0167146. doi:10.1371/ journal.pone.0167146

47. Bager P, Hvas CL, Dahlerup JF. Drug specific hypophosphatemia and hypersensitivity reactions following different intravenous iron infusions. Br J Clin Pharmacol. 2017;83(5):1118-1125. doi:10.111 1/bcp.13189

48. Stöhr R, Sandstede L, Heine GH, Marx N, Brandenburg V. High-dose ferric carboxymaltose in patients with HFrEF induces significant hypophosphatemia. J Am Coll Cardiol. 2018;71(19):2270-2271. doi:10.1016/j.jacc.2018.03.448

49. Sari V, Atiqi R, Hoorn EJ, Heijboer AC, van Gelder T, Hesselink DA. Ferric carboxymaltose-induced hypophosphataemia after kidney transplantation. Neth J Med. 2017;75(2):65-73.

50. Anand G, Schmid C. Severe hypophosphataemia after intravenous iron administration. BMJ Case Rep. 2017;2017:bcr2016219160. doi:10.1136/bcr-2016-219160

51. Blazevic A, Hunze J, Boots JMM. Severe hypophosphataemia after intravenous iron administration. Neth J Med. 2014;72(1):49-53.

52. Fierz YC, Kenmeni R, Gonthier A, Lier F, Pralong F, Coti Bertrand P. Severe and prolonged hypophosphatemia after intravenous iron administration in a malnourished patient. Eur J Clin Nutr. 2014;68 (4):531-533. doi:10.1038/ejen.2014.20
53. Klein K, Asaad S, Econs M, Rubin JE. Severe FGF23-based hypophosphataemic osteomalacia due to ferric carboxymaltose administration. BMJ Case Rep. 2018;2018:bcr-2017-222851. doi:10. 1136/bcr-2017-222851

54. Mani L-Y, Nseir G, Venetz J-P, Pascual M. Severe hypophosphatemia after intravenous administration of iron carboxymaltose in a stable renal transplant recipient. Transplant. 2010;90(7):804-805. doi:10.10 97/TP.0b013e3181f00a18

55. Moore KLF, Kildahl-Andersen O, Kildahl-Andersen R, Tjønnfjord GE. Uncommon adverse effect of a common medication. Tidsskr nor Laegeforen. 2013;133(2):165. doi:10.4045/tidsskr.12.0494

56. Urbina T, Belkhir R, Rossi G, et al. Iron supplementation-induced phosphaturic osteomalacia: FGF23 is the culprit. J Bone Miner Res. 2018;33(3):540-542. doi:10.1002/jbmr.3369

57. Vandemergel X, Vandergheynst F. Potentially life-threatening phosphate diabetes induced by ferric carboxymaltose injection: a case report and review of the literature. Case Rep Endocrinol. 2014; 2014:843689. doi:10.1155/2014/843689

58. Vasquez-Rios G, Marin E. Harder to breathe: an unusual case of severe hyperphosphaturic hypophosphatemia and normal FGF-23 levels in a young female patient. Am J Kidney Dis. 2018;71:594.

59. Bansal VK. Serum Inorganic Phosphorus. Butterworths; 1990. Available from: http://www.ncbi.nlm.nih.gov/pubmed/21250152. Accessed July 10, 2019.

60. Huang LL, Lee D, Troster SM, et al. A controlled study of the effects of ferric carboxymaltose on bone and haematinic biomarkers in chronic kidney disease and pregnancy. Nephrol Dialysis transplant. 2018;33(9):1628-1635. doi:10.1093/ndt/gfx310

61. Schouten BJ, Hunt PJ, Livesey JH, Frampton CM, Soule SG. FGF23 elevation and hypophosphatemia after intravenous iron polymaltose: a prospective study. J Clin Endocrinol Metab. 2009;94(7):2332-23 37. doi: $10.1210 /$ jc. $2008-2396$

62. Wolf M, Chertow GM, Macdougall IC, Kaper R, Krop J, Strauss W. Randomized trial of intravenous iron-induced hypophosphatemia. JCI Insight. 2018;3:23. doi:10.1172/JCI.INSIGHT.124486

63. Wolf M, Rubin J, Achebe M, et al. Effects of iron isomaltoside vs ferric carboxymaltose on hypophosphatemia in iron-deficiency anemia: two randomized clinical trials. JAMA. 2020;323(5):432-443. doi:10.1001/jama.2019.22450
Therapeutics and Clinical Risk Management

\section{Publish your work in this journal}

Therapeutics and Clinical Risk Management is an international, peerreviewed journal of clinical therapeutics and risk management, focusing on concise rapid reporting of clinical studies in all therapeutic areas, outcomes, safety, and programs for the effective, safe, and sustained use of medicines. This journal is indexed on PubMed Central, CAS

\section{Dovepress}

EMBase, Scopus and the Elsevier Bibliographic databases. The manuscript management system is completely online and includes a very quick and fair peer-review system, which is all easy to use. Visit http://www.dovepress.com/testimonials.php to read real quotes from published authors. 